\title{
Hybrid Compression Algorithm for Wireless Sensor Network
}

\author{
Amr M. Kishk, Nagy W. Messiha, Nawal A. Elfishawy, Abdelrahman A. Elkafs, and Ahmed H. Madian
}

\begin{abstract}
Discrete Wavelet Transform (DWT) was developed to improve the performance of data compression. Hybrid Fast Zonal-DCT with DWT is proposed to enhance the image compression during the transmission in WSN. The reduction in power consumption relies on data distribution in the clusters. The performance of the proposed algorithm is monitored by some metrics. The enhancement in image quality and compression ratio is achieved.
\end{abstract}

Index Terms-DWT compression, zonal-DCT compression, image quality, processing time.

\section{INTRODUCTION}

Wireless Sensor Network (WSN) is composed of multiple battery-operated nodes .These nodes consist of a Micro Electro Mechanical System, a low-power Digital Signal Processor, a radio frequency circuit, and a battery [1]. WSN can be used in multiple fields such as :remote monitoring and object-tracking in military applications, temperature measurement of different environments, radiation detection in the nuclear reactors, and wide range of applications in different fields. There are many constraints that should be considered to design sensor nodes to use them in some applications. One of these constraints is energy consumption which specify the life time of node battery [2]. The energy consumption due to transmitted data should be limited. The reduction in the data size by compression reduces the transmission power. However, it is crucial to select an image compression algorithm, which requires less memory access during execution time.

JPEG is one of the image compression algorithms [3]. It depends on Discrete Cosine Transform (DCT), Quantization, and Entropy Encoding, while its decoder stages are the inverse of the JPEG encoder stages, as in Fig. 1. The N-bits 1-D DCT can be expressed as in (1) [4]. Based on the related works about fast DCT algorithms, Fast Zonal DCT is proposed in [5]. In Fast Zonal coding, the only coefficients within a specified region are encoded. Refer to equation (1), the number of operations is reduced to 9 multiplications and 24 additions, and it allows an efficient turning of the trade-off between energy consumption and image distortion. DCT is

Manuscript received November 20, 2013; revised January 6, 2014. This work was supported in part by Nuclear Research Centre members of Egyptian Atomic Energy Authority (EAEA) and Faculty members of Faculty Electronics Engineering (FEE), Egypt.

Amr M. Kishk, Abdelrahman A. Elkafs, and Ahmed H. Madian are with Egyptian Atomic Energy Authority, Cairo 13759, Egypt (e-mail: amr.kishk@yahoo.com, alkafas@yahoo.com, ah_madian@hotmail.com).

Nagy W. Messiha and Nawal A. Elfishawy are with the Faculty of Electronics Engineering, Elmounfya University, Menouf 32951, Egypt (e-mail: dr.nagy_wadie@hotmail.com,nelfishawy@hotmail.com). replaced by DWT to improve the image compression. So JPEG was under name JPEG2000. The description of JPEG2000 in WSN is shown in next section.

$$
F(k)=\frac{C(k)}{2} \sum_{x=0}^{7} f(k) \cos \left(\frac{2 x+1}{16} k \pi\right), k=0, \ldots, 3
$$

where: $C(k)=\left\{\begin{array}{cl}\sqrt{1 / 2} & , k=0 \\ 1 & , k>0\end{array}\right.$

The goal of this study is to enhance the image compression in JPEG2000. The hybrid of fast Zonal-DCT (at $k=0, \ldots, 3$ ) with DWT in 1-D and 2-D is the main target of this paper. The benefits of hybrid both algorithms is getting higher compression rate with high decompressed image quality is shown in this paper.

This paper is organized as follows :Section II introduces survey on JPEG2000 compression, Section III shows the hybrid algorithm, and Sections IV and Section V give the results and conclusions.

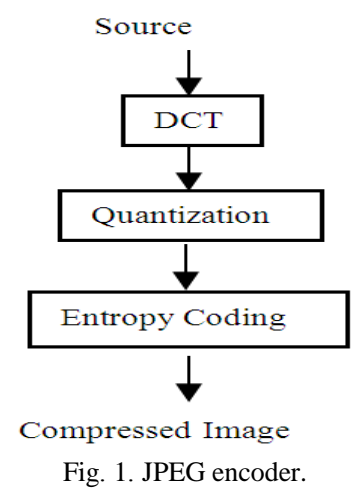

\section{JPEG2000 COMPRESSION}

JPEG2000 encoder consists of three stages: DWT, Quantization, and Entropy Encoding. The basic idea of the distributed JPEG2000 image compression is the distribution of the DWT workload to several nodes in the same cluster [3]. The data is partitioned into $n$ blocks $R_{1}, \ldots, R_{n}$ in the cluster centre to select number of nodes from its cluster region, equals to the number of blocks $R_{i}, i=1,2, \ldots, n$, to perform 1-D wavelet (1-D DWT) on $R_{i}$; one block per node. Once the 1-D DWT is done on rows, the cluster center collects the intermediate results $P_{1}, \ldots, P_{n}$ to send them to the next cluster centre to apply the same operations but on columns instead of rows to have 2-D DWT as a final result as shown in Fig. 2. The Quantization and the Entropy Encoding are performed at the sink node. In the Quantization Stage, each pixel is divided by a quantization step, Q, as in [3]. Entropy Encoding uses the 
Huffman code to generate codes to send them to the destination instead of the quantized value. The decoding process is the inverse of the encoding one.

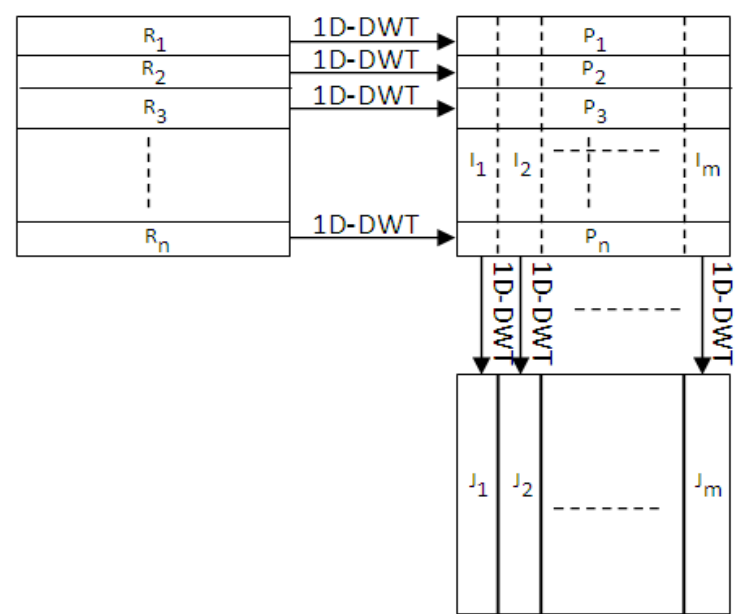

Fig. 2. 2-D DWT processing.

\section{Proposed Hybrid AlgORITHM}

The addition of Zonal-DCT (at $k=0, \ldots, 3$ ) between DWT and Quantization stage is the main idea of the proposed hybrid algorithm in 1-D or 2-D as in Fig. 3. 1-D DWT Zonal means the hybrid 1-D DWT with 1-D Zonal (at $k=0, \ldots, 3$ ) while 2-D DWT-Zonal means the hybrid 2-D DWT with 2-D Zonal DCT (at $k=0, \ldots, 3$ ).

Because of the problem of power consumption, the image compression process is partitioned between the sensors. 1-D Zonal DCT is processed in the same cluster. 2-D DWT, 2-D Zonal, and 1-D DWT-Zonal are processed in two adjacent clusters. 2-D DWT-Zonal is processed in four adjacent clusters.



Fig. 3. Block diagram of proposed hybrid algorithm.

\section{RESULTS AND DISCUSSIONS}

The proposed hybrid algorithm, Zonal-DCT (at $k=0, \ldots, 3$ ), and JPEG2000 are compared with each other in their performance using MATLAB .Mandrill image of size $512 \times 512$ is the original image in bmp format used for comparison purpose. The comparison is applied between

\section{- 2-D DWT}

It means applying 1-D DWT on rows followed by applying 1-D DWT on columns.
- 1-D Zonal-DCT (at $k=4$ )

It means applying fast zonal algorithm on image rows, and $k=0, \ldots, 3$.

- 2-D Zonal-DCT (at $k=4$ )

It means applying 1-D Zonal-DCT on rows followed by applying 1-D Zonal-DCT on columns, and $k=0, \ldots, 3$.

- Hybrid 1-D DWT-Zonal (at $k=4$ )

It means applying 1-D DWT on image rows followed by applying 1-D Zonal-DCT on resulted image rows, and $k=0, \ldots, 3$.

- Hybrid 2-D DWT-Zonal (at $k=4$ )

It means applying 2-D DWT on the image followed by applying 2-D Zonal-DCT on the resulted image, and $k=0, \ldots, 3$.

The benefits of the Zonal DCT (at $k=4$ ) after DWT are the reduction for the number of multiplications used to compress the image and getting high quality of reconstructed image with high compression rate. Table I shows that the number of multiplications reduced by $50 \%$ and $75 \%$ in the case of 1-D DWT-Zonal and 2-D DWT-Zonal respectively. Table II shows the enhancement in compression rate of hybrid 2-D DWT-Zonal than the others. The compression rate scores 1:16 in the case of Hybrid 2-D DWT-Zonal while 1:4 in the case of 2-D DWT.

TABLE I: NUMBER OF MULTIPLICATIONS AND ADDITIONS USED BY ZONAL

\begin{tabular}{|lcc|}
\hline & 1-D Zonal & 2-Zonal \\
\hline № of MUL without hybrid & 294912 & 442368 \\
№ of MUL with hybrid & 147456 & 110592 \\
Reduction in № of MUL(\%) & $50 \%$ & $75 \%$ \\
№ of ADD without hybrid & 786432 & 1179648 \\
№ of ADD with hybrid & 393216 & 294912 \\
Reduction in № of ADD (\%) & $50 \%$ & $75 \%$ \\
\hline
\end{tabular}

\begin{tabular}{|c|c|c|c|c|c|}
\hline Compression & 2 & $1 \mathrm{D}$ & $2 \mathrm{D}$ & $1 \mathrm{D}$ & D \\
\hline Tyno & DWT & $70 \mathrm{l}$ & 7 ond & DUT Zon & DUT 7on \\
\hline 1уре & DW 1 & Zonal & Zonal & DW T-Zonal & DWT-Zonal \\
\hline $\begin{array}{c}\text { Compression } \\
\text { rate }\end{array}$ & $1: 4$ & $1: 2$ & $1: 4$ & $1: 4$ & $1: 16$ \\
\hline
\end{tabular}

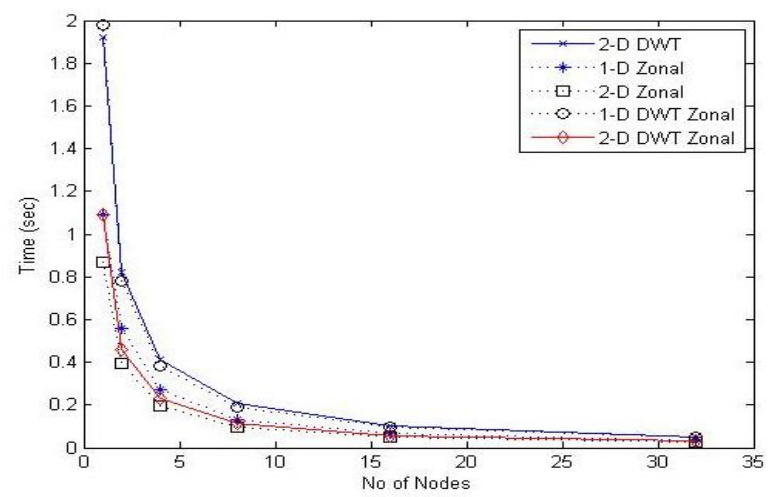

Fig. 4. Processing time.

The distribution of data processing between nodes reduces the power consumption per node. The total processing time is compared with the number of nodes used for processing per cluster as shown in Fig. 4. The comparison shows that 2-D Zonal-DCT takes the minimum processing time while 
2-D-DWT-Zonal scores the next one. The other comparison metrics are Peak Signal to Noise (PSNR) [3], Spatial Frequency (SF) [6], and Correlation Coefficient (CC) [7]. The comparison shows that:

- The proposed hybrid 2-D DWT-Zonal gives higher PSNR for high Q while 2-DWT for low Q as shown in Fig. 5.

- The measured CC and SF for the proposed hybrid 2-D DWT-Zonal gives good results between the different algorithms as shown in Fig. 6 and Fig. 7 respectively.

- The previous results ensure the preference of 2-D DWT-Zonal than the others.

The benefit of data compression in WSN is the reduction in power consumption at the transmission. Proposed hybrid 2-D DWT-Zonal DCT enhances the compression rate and PSNR for high $Q$. The distribution of data compression in between sensors reduced the power consumption .The comparisons shows the preference of proposed hybrid 2-D DWT-Zonal than the others.

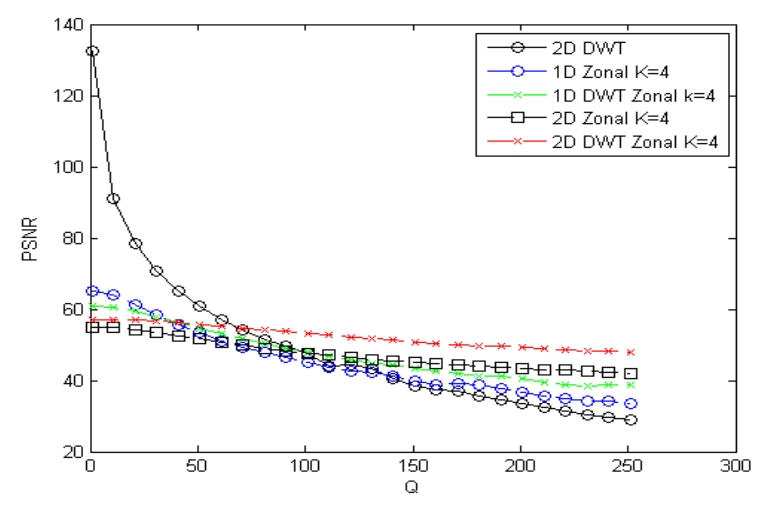

Fig. 5. PSNR comparison.



Fig. 6. CC comparison.



\section{REFERENCES}

[1] S. Hussain, M. Razzak, A. Minhas, M. Sher, and G. Tahir, "Energy efficient image compression in wireless sensor networks," International Journal of Recent Trends in Engineering , vol. 2, no. 1, 2009.

[2] M. Dawood, L. Ahila, S. Sadasivam, and G. Athisha, "Image compression in wireless sensor networks- a survey," International Journal of Applied Information Systems, vol. 1, no. 9, February 2012.

[3] W. Huaming and A. Alhussein, "Energy efficient distributed image compression in resource-constrained multihop wireless networks," Computer Communications, vol. 28, no. 14, pp. 1658-1668, 2005.

[4] R. Conzalez and R. Woods, Digital Image Processing, Upper Saddle River, New Jersey: Prentice-Hall, Inc., 2001.

[5] L .Makkaoui, V .Lecuire, and J .Moureaux, "Fast zonal DCT-based image compression for wireless camera sensor networks," in Proc. 2nd International Conference on Image Processing Theory, Tools and Applications, IPTA, 2010, pp. 126-129.

[6] Y. Zheng, E. Essock, B. Hansen, and A. Haun, "A new metric based on extended spatial frequency and its application to DWT based fusion algorithms," Information Fusion, vol. 8, no. 2, pp. 177-192, 2007.

[7] N. El-Fishawy and O. Zaid, "Quality of encryption measurement of Bitmap images with RC6, MRC6, and Rijndael block cipher algorithms," International Journal of Network Security, vol. 5, no. 3, pp. 241-251, November 2007.

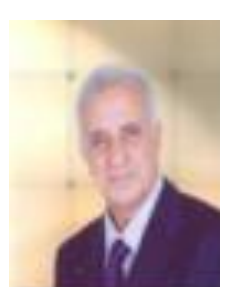

Nagy Wadie Messiha received the B.S. in Electrical Engineering Telecommunication Department, Ein Shams University, Cairo, Egypt, June 1965, and M.S. in telecommunication engineering, Helwan University, Cairo, Egypt, 1973, and the german (Dipl. Ing.) and (Dr. Ing.) from University of Stuttgart, in 1978 and 1981 respectively. From 1981 to 1987 . Currently, he is a professor at the Department of Communication Engineering, Menoufia University, Menouf, Egypt. His research interested is traffic modeling and performance enhancement in communication and computer networks, cognetive networks, and network security.

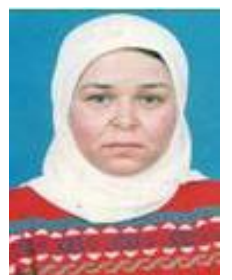

Nawal El-Fishawy received the $\mathrm{PhD}$ degree in mobile communications in the faculty of Electronic Eng., Menoufia University, Menouf, Egypt, in collaboration with Southampton University in 1991. Now she is a professor at Computer Science and Engineering Dept., Faculty of Electronic Eng. Her research interest includes computer communication networks with emphasis on protocol design, traffic modeling and performance evaluation of broadband networks and multiple access control protocols for wireless communications systems and networks. Now she directed her research interests to the developments of security over wireless communications networks (mobile communications, WLAN, Bluetooth), VOIP, and encryption algorithms.

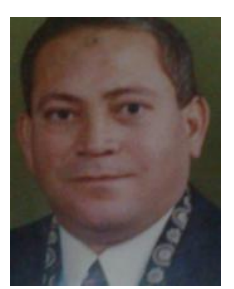

Abdelrahman A. Elkafas received the B.S. in Nuclear engineering, Alexandria University, alexandria, Egypt, June 1980, and M.S. in analysis of residual heat removal systems in PWR-NPPs, Alexandria University, Alexandria, Egypt, June 1990, and Ph.D in safety analysis of an expert reactor protection system in PWR-NPPs, Alexandria University, Alexandria, Egypt, June 1996. He was an associate professor in 2002, Nuclear Research Centre, Egypt. Currently, he is a professor in the Nuclear Research Centre, Egypt. He is interested in safe operation of nuclear reactors, automatic control of research reactors, and physical protection systems (design and evaluation).

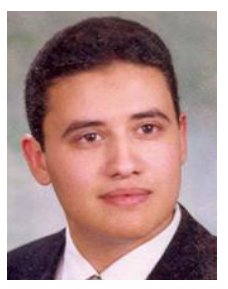

Ahmed H. Madian was born in 1975. He received the B.Sc. degree with honors, the M.Sc., and the Ph.D. degrees in electronics and communications from Cairo University, Cairo, Egypt, in 1997, 2001, and 2007, respectively. $\mathrm{He}$ is currently an associate professor in the Electronics Engineering Department, Micro-Electronics Design Center, Egyptian Atomic Energy Authority, and Cairo, Egypt. Dr. Madian 
served as assistant professor in the Electronics Engineering Department, Faculty of Information Engineering and Technology, German University in Cairo (GUC) from 2008 till now. Dr. Madian is the co-author of 20 research papers in different scientific journals and has served as program and publication chair for many conferences. He is a senior member of IEEE and co-founder for the IEEE Robotics Chapter- Egypt section (best chapter on Region 8 for 2013). His research interests are in circuit theory; low-voltage analog CMOS circuit design, current-mode analog signal processing, digital VLSI, system security and mixed/digital applications on field programmable gate arrays.



Amr M. Kishk was born in Ashmun city, Egypt, on July 11,1981 . He received the B.S. in electronics and electrical communication engineering, Menoufia University, Menouf, Egypt, 2003, and M.S. in data security in wireless local area network, Menoufia University, Menouf, Egypt, 2010. Now, he works as a lecturer assistant in Egyptian Atomic Energy Authority (EAEA), Cairo, Egypt. His research interests include wireless sensor network (WSN), data security in wireless network. 